\title{
Study on the Modal Analysis Method of Two Screw Pump Rotor
}

\author{
Zhang Fan, ${ }^{\mathrm{a},}$, Zhu Jun ${ }^{\mathrm{a}}$, Wu Gaojie ${ }^{\mathrm{a}}$, Sun Yajing ${ }^{\mathrm{a}}$ \\ ${ }^{a}$ Mechanical Engineering College, Yangzhou University, Yangzhou, China \\ *Corresponding Author: fzhang@yzu. edu. cn
}

\begin{abstract}
In order to avoid the occurrence of the resonance of the twin screw pump under the periodic change of the fluid, the mechanical performance of the twin screw pump is optimized. To simplify the screw rotor, the vibration mechanical model that the screw rotor, using theoretical methods to solve the first order natural frequency is $525.9835 \mathrm{~Hz}$, then using Ansys software to carry out modal analysis under different constraint conditions on the screw rotor, the modal that the screw rotor, the finite element modal analysis of the screw rotor in spring damping under constraint the $526.4 \mathrm{~Hz}$ theory and the results of software calculation error of only $0.08 \%$, prove the accuracy of the finite element modal damping spring constraint model of screw rotor modal calculation, the results can provide a basis for optimization of mechanical properties of double screw pump.
\end{abstract}

Keywords: twin screw pump, screw rotor, modal analysis, ANSYS Workbench.

\section{Introduction}

Double screw pump is a fluid driven device, its core components are a couple of intermeshing screw rotor, with high-speed rotation of the screw, complete inhalation, transport and discharge of the three continuous cycle of process fluid, fluid driven transport task [1]. In the design phase of the rotors, in addition to considering the factors of screw strength, dimensional accuracy, tolerance, also need to assess the dynamic characteristics in the actual operation process, especially the occurrence of periodic fluid may screw rotor periodic changes in the strong resonance, which will directly affect the normal operation of double the screw pump, this paper through the ANSYS software Workbench for finite element analysis of screw constraint modal analysis, the natural frequency and vibration mode. In view of the screw rotor supported by tapered roller bearings, the constrained modal analysis to coincide with the actual constraint state, this paper adopts different constraint models to simulate the actual bearing constraints, through the analysis and comparison of the natural frequency and vibration mode, obtain more accurate analysis results to avoid the resonance of double screw pump at work. In order to achieve the purpose of optimization design of screw rotor performance.

\section{Theoretical Calculation of Natural Frequency of Twin Screw Pump Rotor}

A mechanical theory on an infinite number of natural frequencies, but in the process of calculation of the natural frequency, the structure is discretized into finite order solution, and calculate the lowest natural frequency of the structure is the first-order natural frequency, which is the fundamental frequency of the structure, it is the most important physical data in practical applications, involving the structure will not suffer some frequency load caused by resonance damage ${ }^{[2]}$. As shown in Figure 1 the structure of the screw rotor, the rotor structure is simplified, the vibration mechanical model is simplified as shown in Figure 2, the screw rotor made of stainless steel, the young's modulus of $\mathrm{E}$ is $2.08 \times 10^{11} \mathrm{~Pa}$, the Poisson's ratio is 0.30 , density is $7800 \mathrm{Kg} / \mathrm{m}^{3}$. The first-order natural frequency using the dunkery calculation method of screw rotor.

The screw rotor shaft is simplified as uniform, equivalent diameter $\mathrm{d}$ by type (1) to calculate $36.6 \mathrm{~mm}, d_{i}$ and $l_{i}$ respectively, the shaft diameter and length of screw rotor; simplified is divided into three parts, bearing between the shaft length is $633 \mathrm{~mm}$, simplified lumped mass M2, shaft bearing left the extended end of the length of $90 \mathrm{~mm}$, simplified lumped mass M1, shaft bearing at the right end of the extended end of the length of $55 \mathrm{~mm}$, simplified 
lumped mass M3.

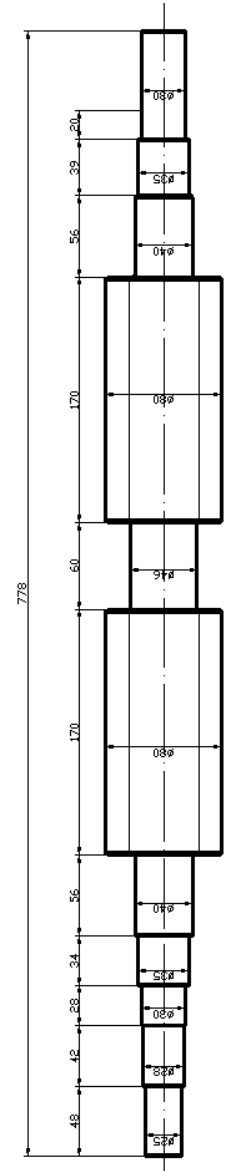

Fig. 1. Screw rotor

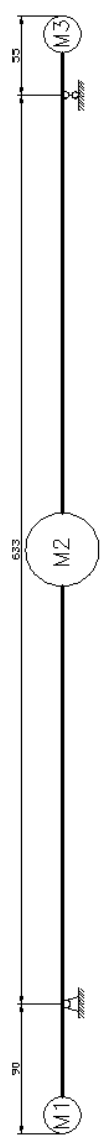

Fig. 2. vibration model of Screw rotor

$$
\mathrm{d}=\sqrt[4]{\frac{L}{\sum_{i=1} \frac{l_{i}}{d_{i}^{4}}}}
$$

List the 3 order differential equation of vibration mechanics model:

$$
\left[\begin{array}{ccc}
m_{1} & 0 & 0 \\
0 & m_{2} & 0 \\
0 & 0 & m_{3}
\end{array}\right]\left[\begin{array}{c}
\ddot{X}_{1} \\
\ddot{X}_{2} \\
\ddot{X}_{3}
\end{array}\right]+\left[\begin{array}{lll}
K_{11} & K_{12} & K_{13} \\
K_{21} & K_{22} & K_{23} \\
K_{31} & K_{32} & K_{33}
\end{array}\right]\left[\begin{array}{l}
X_{1} \\
X_{2} \\
X_{3}
\end{array}\right]=0
$$

The $\mathrm{m}_{1}, \mathrm{~m}_{2}$ and $\mathrm{m}_{3}$ are used to simplify the three sections of the concentrated mass, $X_{1}, X_{2}$ and $X_{3}$ for the response of the two transverse degrees of freedom, $\mathrm{K}_{\mathrm{ii}}$ is the stiffness coefficient.

The characteristic frequency equation of the reduced system is obtained by solving the differential equation:

$\left|-\frac{1}{\omega^{2}}\left[\begin{array}{lll}1 & 0 & 0 \\ 0 & 1 & 0 \\ 0 & 0 & 1\end{array}\right]+\left[\begin{array}{lll}a_{11} & a_{12} & a_{13} \\ a_{21} & a_{22} & a_{23} \\ a_{31} & a_{32} & a_{33}\end{array}\right]\left[\begin{array}{ccc}m_{1} & 0 & 0 \\ 0 & m_{2} & 0 \\ 0 & 0 & m_{3}\end{array}\right]\right|=0$

Where $\omega$ is the natural frequency, $a_{i i}$ flexibility coefficient.

The expansion (3), get the $\mathrm{n}$ polynomial of $\frac{1}{\omega^{2}}$, let the formula $\left(\frac{1}{\omega^{2}}\right)^{n-1}$ have the same coefficient:

$$
\frac{1}{\omega_{1}^{2}}+\frac{1}{\omega_{2}^{2}}+\cdots+\frac{1}{\omega_{n}^{2}}=\sum_{i=1}^{n} a_{i i} m_{i}
$$

Based on the assumption that the higher order frequency is much larger than the first natural frequency of the rotor, the formula (4) can be reduced to the equation of the first order natural frequency

$$
\frac{1}{\omega_{1}^{2}} \approx \sum_{i=1}^{n} a_{i i} m_{i}
$$

The first natural frequency of the rotor can be calculated by formula (5) $525.9835 \mathrm{~Hz}$

\section{Modal Analysis Pretreatment of Screw Rotor}

Modal analysis is mainly used to determine the vibration characteristics of the structure, but also other dynamics analysis, this paper adopts the finite element modal analysis, finding and establishing appropriate model for modal analysis of the screw of each order natural frequency and vibration mode, the first-order natural frequency of the request can be consistent with the theoretical calculation, so as to reduce the manual calculation by using the finite element modal analysis for theoretical calculation. The analysis flow chart is shown in figure 3:

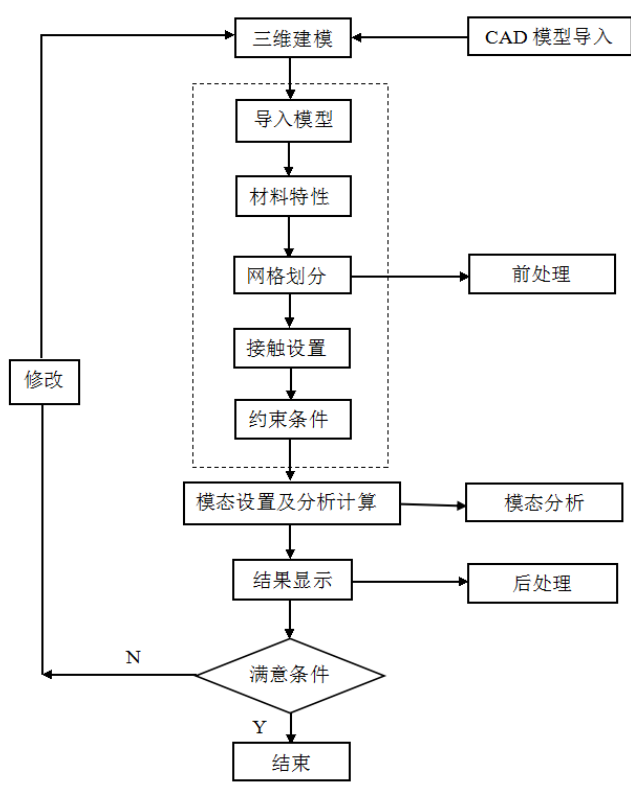

Fig. 3 flow chart of screw rotor modal analysis

\subsection{Entity modeling and mesh generation}


A finite element model of the screw should reflect the geometric structure and mechanical properties of screw screw, but small chamfer, keyway and other small features a very small unit size in finite element mesh, the local unit number is too large, leading to increase the amount of calculation, or even make the calculation and analysis of failure. These small features has little influence on the modal, so the mesh before pretreatment is needed and can be simplified, ignoring the impact analysis of smaller features on mode, to ensure that the structure of the finite element mesh grid density distribution, moderate [3].

This paper selects the screw rotor profile by epicycloids plus cycloid combining form, structure parameters are shown in table 1.

Table 1 dimensional model parameters of screw

\begin{tabular}{|l|l|l|l|l|}
\hline name & $\begin{array}{l}\text { Number } \\
\text { of tooth }\end{array}$ & $\begin{array}{l}\text { Root } \\
\text { radiu } \\
\mathrm{s} \\
(\mathrm{mm})\end{array}$ & $\begin{array}{l}\text { Tip } \\
\text { radius } \\
(\mathrm{mm})\end{array}$ & $\begin{array}{l}\text { Pitch } \\
\text { screw } \\
\text { length( } \\
\mathrm{mm})\end{array}$ \\
\hline $\begin{array}{l}\text { Param } \\
\text { eter }\end{array}$ & 30 & 50 & 100 & 778 \\
\hline
\end{tabular}

Considering the convenient and intuitive CAD interface file and ANSYS to support a variety of factors such as the complexity of CAD screw, system modeling, this paper adopts the three-dimensional modeling software UG modeling of screw rotor, and then imported into the finite element software for the corresponding treatment.

Because the screw part of the screw type line is swept along the spiral form, and belongs to the key parts on the grid points, to put it and the adjacent part of a separate division, set up small grid size, the size of a higher level intelligence division, the remaining part of the mesh size and level can not be too high, otherwise it will affect the speed and accuracy analysis. In view of hexahedral mesh may complex model cannot be divided, the tetrahedral mesh of the screw rotor, and the spiral part is encrypted, the finite element model of screw rotor after meshing as shown in Figure 4, the total number of nodes is 921429, a total of 3928168 units.

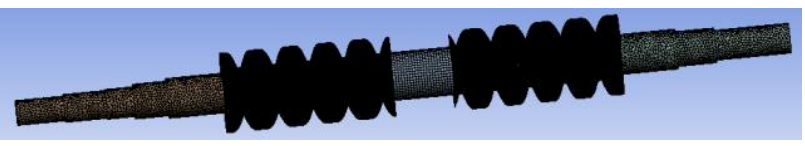

Fig. 4 finite element model of screw rotor

The quality of the grid has a very important impact on the accuracy of the modal analysis, after the grid division of the general Element quality (mesh quality) and Skewness (skewness) of these two aspects of the inspection [5]. Element quality is the ratio of the volume and length of the grid unit, between 0 and 1,0 of the worst, the best 1 , figure 5 is the unit grid quality map, it can be seen from the figure is less than 0.5 of the few, the vast majority are more than 0.75 , the overall quality of the said grid division is very good; Figure 6 the quality of unit slope inclination on the map, the mesh quality is also very important, its value is between 0 and 1, 0, 1 is the worst and the best in 0.96 , we can see from Figure 4 is greater than the number of grid 0.5 is very small, most of them are below 0.38 . All of the above two aspects can be obtained: the overall network is suitable for modal analysis.

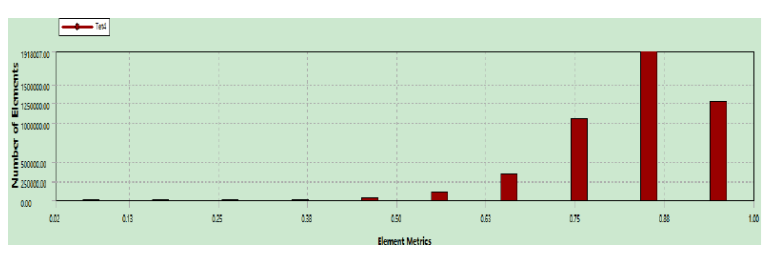

Fig. 5 quality check of grid unit - Element quality

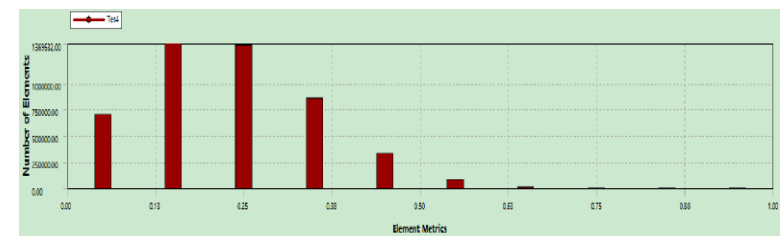

Fig. 6 grid unit quality check - Skewness

\section{2 applying boundary settings and constraints}

When carrying out the modal analysis of the three-dimensional entity model, entity model of the natural frequency and vibration mode of the structure and only the stiffness characteristics (including elastic modulus, boundary conditions and geometry) and mass distribution, the force has little effect on the natural frequency of the screw, the key factors affecting the results of modal analysis is real the type of constraint and mass distribution. In this paper, the study of the twin screw pump in the actual working conditions of the support is one end of a fixed, a walk, and at the end of the screw rotor bearing radial load. Therefore, in the modal analysis of the screw rotor, the position of the screw at both ends of the screw is subjected to the torque, and the circumferential direction is restrained. In the screw installation of bearing parts, this paper adopts the constraint of a cylinder, the elastic support constraint and spring damping constraint three different constraint methods were analyzed and compared by calculation: in the elastic support constraint stiffness is about; in the spring damping constraint stiffness damping is about, is about 12600 . 


\section{Analysis of the Results of Modal Analysis of Screw Rotor}

By means of modal analysis, the natural frequency and vibration mode of the rotor can be calculated, which can prevent the external exciting frequency from the natural frequency when the design and improvement are improved. In view of the low order vibration mode plays a decisive role in the dynamic characteristics of the structure, so the modal analysis is usually the first 6 orders can be [4]

\subsection{Modal Analysis of Cylindrical Surface}

Applying the constraint of cylinder screw in the installation of the bearing parts (i.e. rigid constraints), the first six order natural frequency and maximum deformation by finite element analysis after the screw are shown in Table 2, corresponding to the six modes as shown in figure 7. Through analysis of the results of 1 order and 2 order natural frequency and vibration type is similar to 4 order and 5 order natural frequency and vibration type are similar, in fact, will be near the natural frequency in a certain order of the structure resonance, which caused a strong change mode; screw the front six modes in order to only third the modal shape of the distortion, the other modes are mainly bending deformation.

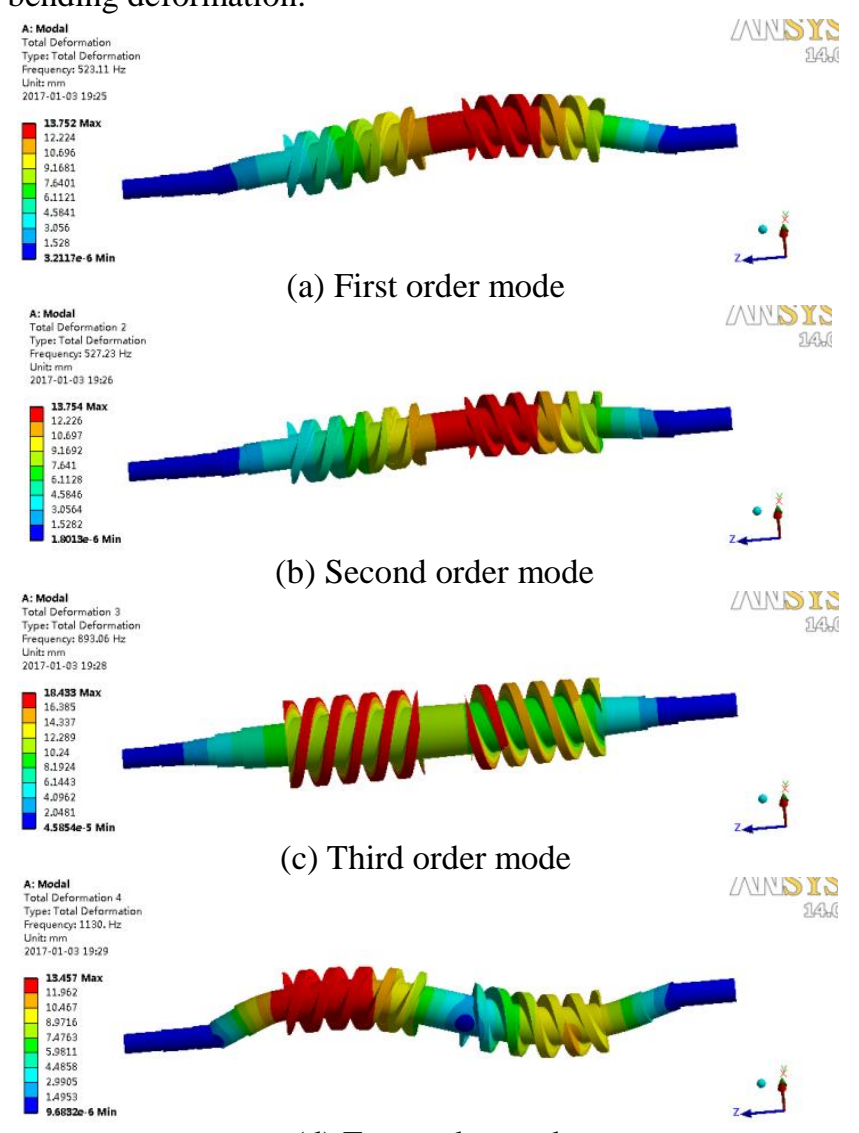

(d) Four order mode

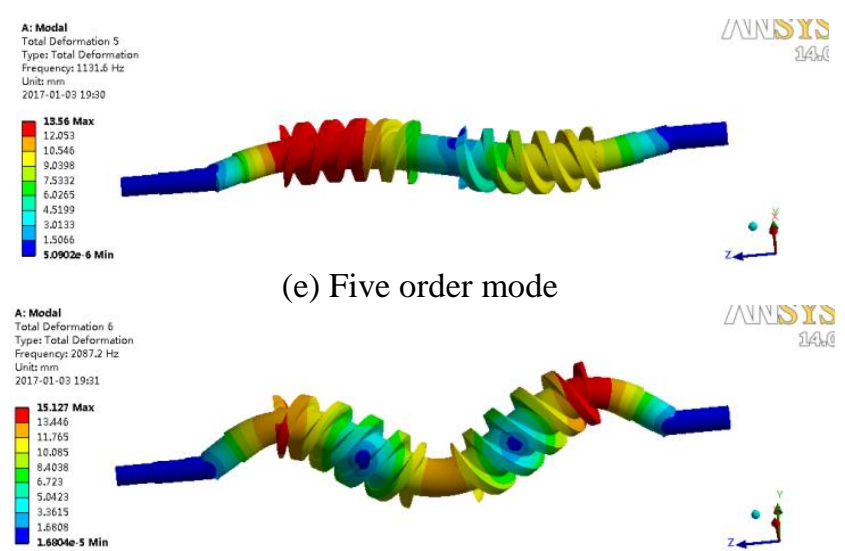

(f) Six order mode

Fig. 6 natural frequencies of each order under the constraint of cylindrical surface

\subsection{Modal analysis of elastic supports}

When the elastic support is applied to the position of the screw bearing, the first six natural frequencies and the maximum deformation of the screw are analyzed by the finite element analysis, as shown in Table 3. The corresponding first six order modes are shown in figure 8. Through analysis of the results of 1 order and 2 order natural frequency and vibration type is similar to 4 order and 5 order natural frequency and vibration mode are similar; the screw top six modes in only third modes to distortion, all other modes are mainly bending deformation.

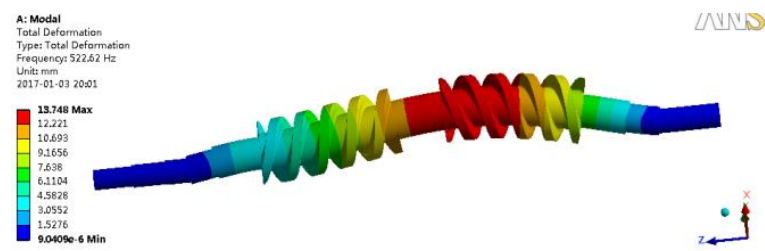

(a) First order mode

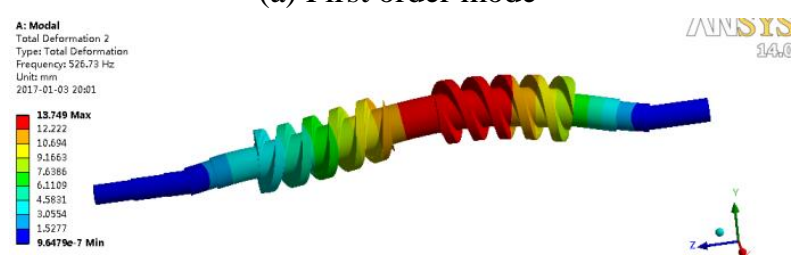

(b) Second order mode

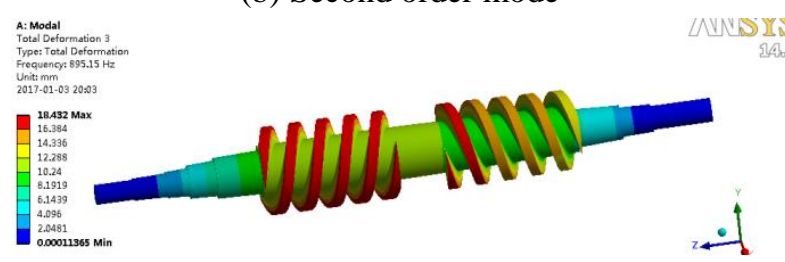

(c) Third order mode

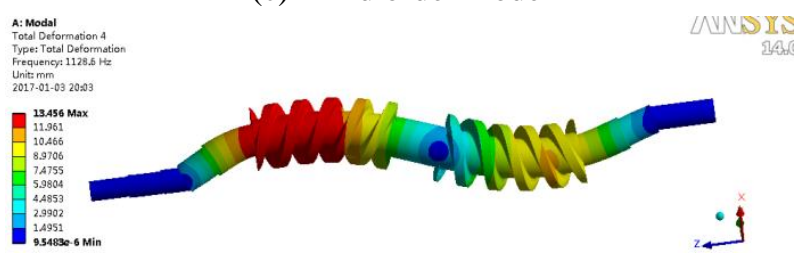

(d) Four order mode 


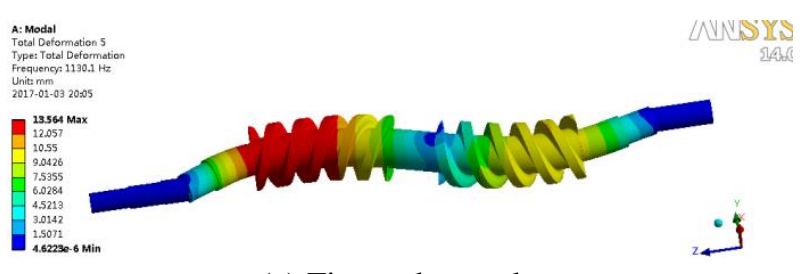

(e) Five order mode

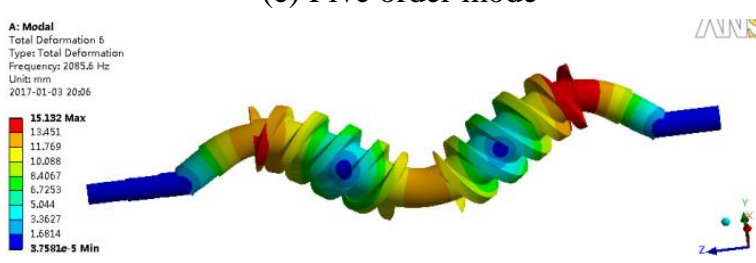

(f) Six order mode

Fig. 8 natural frequencies of each order under the elastic support

\subsection{Modal analysis of spring damper}

Applying the spring damping constraint in the screw installation of the bearing parts, can be simplified as the radial compression spring spring, each bearing elastic support by four uniform composition, each spring stiffness is about $3.57 \times 10^{6} \mathrm{~N} / \mathrm{m}$, damping is about 12600 . Through the finite element analysis, the first six natural frequencies and the maximum deformation of the screw are shown in Table 4, and the corresponding first six order modes are shown in figure 9 . Through analysis of the results of 1 order and 2 order natural frequency and vibration type is similar to 4 order and 5 order natural frequency and vibration mode are similar; the screw top six modes in only third modes to distortion, all other modes are mainly bending deformation.

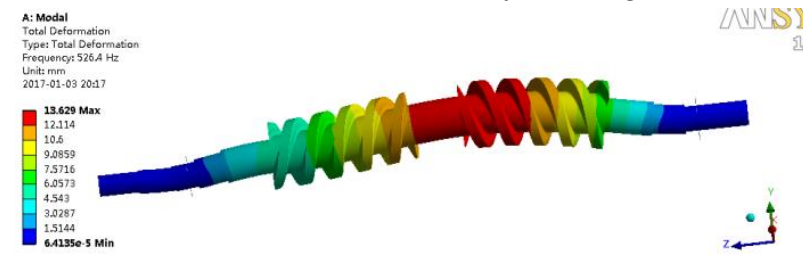

(a) First order mode

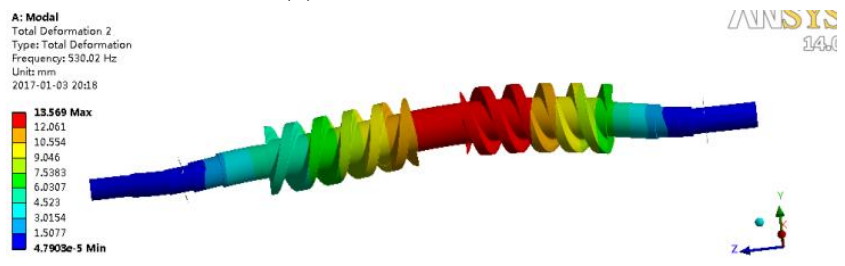

(b) Second order mode

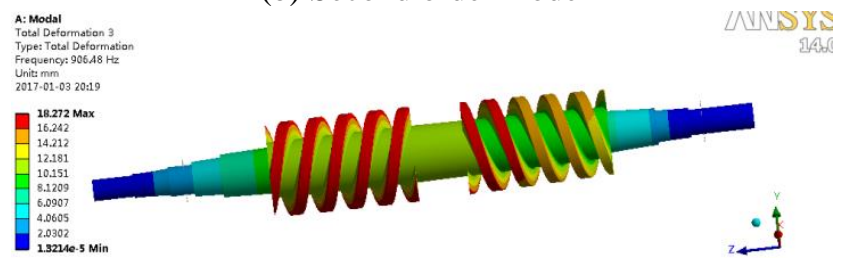

(c) Third order mode

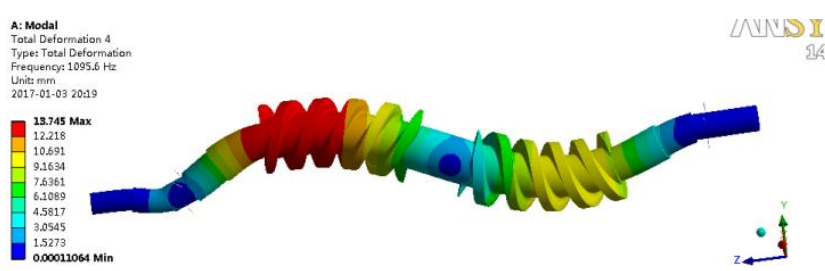

(d) Four order mode

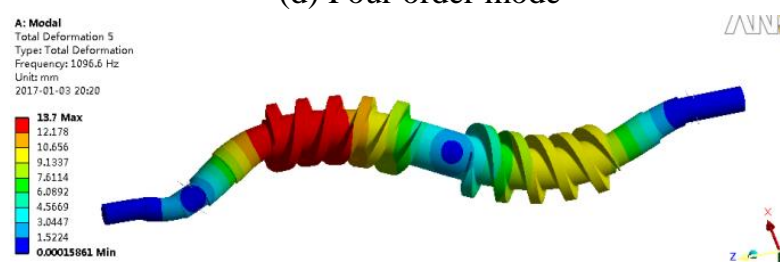

(e) Five order mode

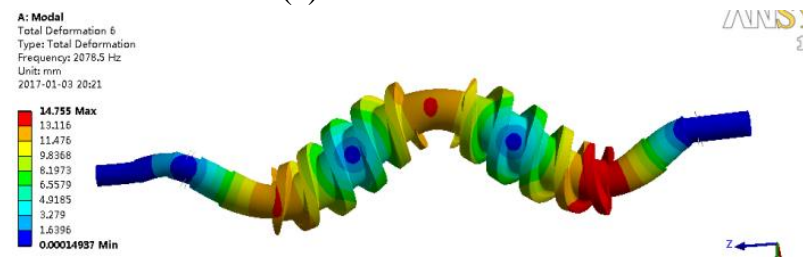

(f) Six order mode

Fig. 9 natural frequency vibration modes of each order under spring damping

\section{4 comparison of modal analysis under different constraints}

In order to compare the difference between the natural frequency and the maximum shape of the screw rotor under different constraints, the relevant data of Table 2, table 3 and table 4 are drawn into a column chart, as shown in Figure 10 and figure 11. Figure 10 analysis: modal analysis the natural frequency are basically the same using cylinder constraint and elastic support constraint in the screw installation of bearings, the difference is not more than $0.5 \mathrm{~Hz}$; and the use of spring damping constraints natural frequency and modal analysis of the first two kind of difference, and the analysis of the first three order the natural frequency is larger than the first two, then three order natural frequency than the previous two cases are small. Figure 11 analysis: modal analysis results of the order maximum deformation is basically the same with the cylindrical surface of the elastic support constraints and constraints in the installation of the bearing screw, a difference of not more than $0.005 \mathrm{~mm}$; the maximum deformation and the use of spring damping constraint modal analysis and the first two cases have large difference, and its analysis the first three order maximum deformation is smaller than the first two, then three order natural frequency than the previous two cases are large. By comparing Figure 7, figure 8 and Figure 9, we can see that the vibration mode of the screw bearing is basically the 
same as that of the cylindrical surface, the elastic support and the spring damper. The conclusions are as follows: in the installation of the bearing screw place respectively using cylindrical modal analysis, elastic support constraint constraint and spring damping constraints, each mode of income is the same, only the use of the natural frequency of the damping spring constraint is different from the maximum deformation.

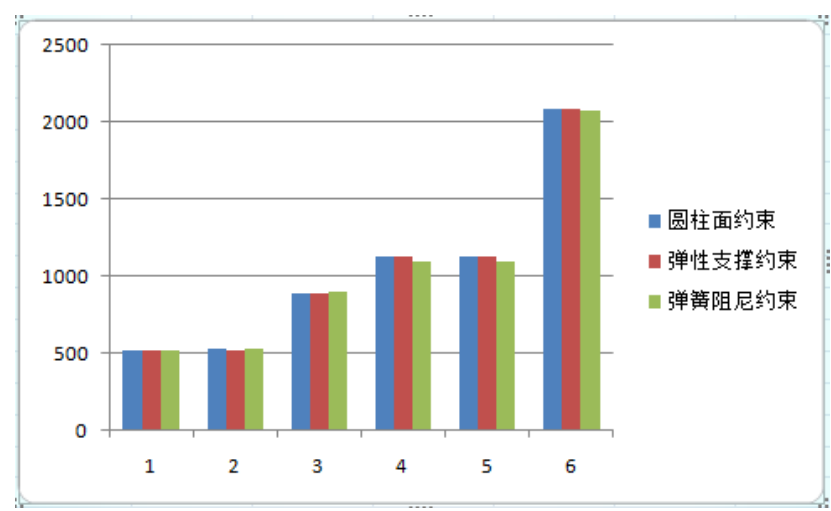

Fig. 10 the natural frequency diagram

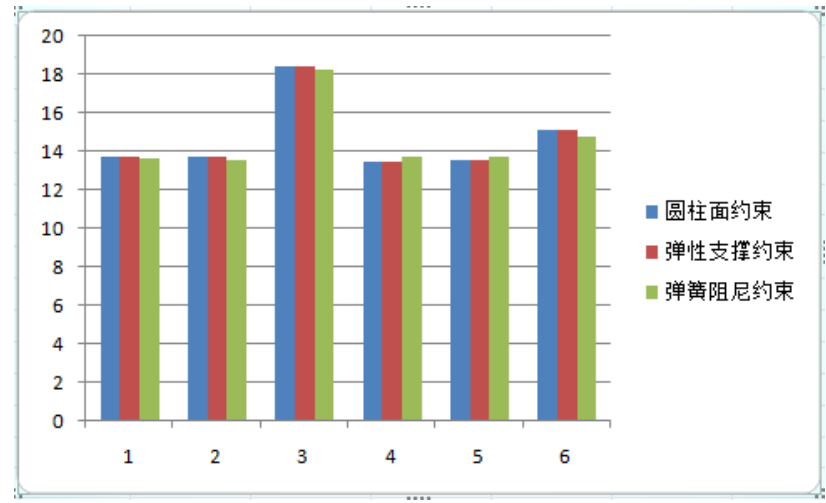

Fig. 11 relation graph of maximal shape variable

\section{Conclusions}

In this paper, through the theoretical calculation of the twin screw pump rotor and the establishment of three kinds of screw restraint model, the finite element modal analysis is made:

(1) using these three different constraint modal analysis consistent mode of income, natural frequency and maximum deformation is different, the natural frequency and the maximum deformation of cylinder constraint and elastic support under the constraint of basically the same, there is a certain difference between the spring damper under the constraint of natural frequency and the maximum deformation and the two kinds of situations;

(2) the theoretical calculation result of the first order natural frequency of the twin screw pump rotor is $525.9835 \mathrm{~Hz}$, and the result of the finite element modal analysis of the screw rotor under the spring damping constraint is $526.4 \mathrm{~Hz}$.

(3) verified by theoretical calculation, proved that the use of spring damping restriction method for screw rotor in finite element modal analysis results more accurate, the use of the modal model of screw rotor analysis can provide the basis for the performance optimization of mechanical double screw pump.

(4) the excitation frequency is far less than the minimum natural frequency of the above three different constraint States, which will not cause resonance..

\section{Acknowledgment}

Thank the China Natural Science Foundation for this project. Item Number: 51375427. Thank the Jiang Su Natural Science Foundation for this project. Item Number: BY2014117-08, BY2015061-04.

\section{References}

(1) Li Futian. Screw pump [M]. Machinery Industry Press, 2010

(2) Gao Shuying, Shen Huoming. Vibration mechanics [M]. Beijing: China Railway Press.2011:51-94.

(3) Chen Xuefeng, Li Bing,. Finite element method and its engineering case $[\mathrm{M}]$. Beijing: Science Press,.2014:162-204. (in Chinese) [3]

(4) Analysis and optimization of the transmission part of large displacement twin screw pump used in ships [D]. Tianjin University of Technology [4].2013.

(5) Xie Guanmo. Vibration mechanics (Second Edition) [M]. Beijing: National Defense Industry Press.2011:94-162.

(6) mamtimin - Aini, Chen Hualei.ANSYS-Workbenc14.0 simulation technology and engineering practice [M]. Beijing: Tsinghua University press.2013:72-75.

(7) ANSYS-Workbenc15.0 analysis of the structure of Lian Tung QuickStart Guide [M]. Beijing: Electronic Industry Press,.2015:153-195.

(8) Wang Linjun, Cao Huiping. Rotor modal analysis based on $[\mathrm{J}]$. ANSYS-Workbench Journal of China Three Gorges University (NATURAL SCIENCE EDITION).2014,36 (6): 89-93.

(9) Luoping, Mu Yang, Liu Heng. Effect of bearing preload on machine tool spindle stiffness $[\mathrm{J}]$. machine tool and hydraulic.2011,39 (17): 9-11.

(10) Wang. Design theory and simulation study of medium 
and high pressure pure water twin screw pump [D]. Chongqing University.2014.

(11) Wu Tengqing, Liu Heng, Jing Minqing. High speed spindle bearing stiffness calculation and modal analysis of mechanical design and manufacture of.2013,8:5-11. [J].

(12) Li Hongguang. Significance of preload of rolling bearing and estimation and adjustment of preload [J]. manufacturing.2004,42 (481): 45-48. 